\title{
ÉDOUARD GLISSANT, Introduzione a una poetica del
} Diverso

\section{Carminella Biondi}

\section{(2) OpenEdition}

\section{Journals}

\section{Édition électronique}

URL : https://journals.openedition.org/studifrancesi/45574

DOI : 10.4000/studifrancesi.45574

ISSN : 2427-5856

Éditeur

Rosenberg \& Sellier

\section{Édition imprimée}

Date de publication : 1 août 2021

Pagination : 417

ISSN : 0039-2944

\section{Référence électronique}

Carminella Biondi, «ÉDouARD glissant, Introduzione a una poetica del Diverso », Studi Francesi [En ligne], 194 (LXV | II) | 2021, mis en ligne le 01 septembre 2021, consulté le 15 octobre 2022. URL : http:// journals.openedition.org/studifrancesi/45574 ; DOI : https://doi.org/10.4000/studifrancesi.45574

Ce document a été généré automatiquement le 15 octobre 2022.

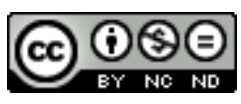

Creative Commons - Attribution - Pas d'Utilisation Commerciale - Pas de Modification 4.0 International - CC BY-NC-ND 4.0

https://creativecommons.org/licenses/by-nc-nd/4.0/ 


\title{
ÉDOUARD GLISSANT, Introduzione a una poetica del Diverso
}

\author{
Carminella Biondi
}

\section{RÉFÉRENCE}

ÉDOUARD GLISSANT, Introduzione a una poetica del Diverso, traduzione di F. Neri, a cura di G.

Sofo, Roma, Meltemi, 2020, 148 pp.

1 Après Le Indie, traduites par Andrea Gazzoni, il faut se réjouir de cette nouvelle édition de la première traduction italienne d'une œuvre de Glissant, faite par Francesca Neri en 1998 et revue par Giuseppe Sofo. Si le poème Les Indes, de 1956, a été défini un texte séminal car il contient en germe tout ce qui va éclore par la suite dans la production de Glissant, Introduction à une poétique du Divers, écrit 40 ans après (1996), peut être considéré comme une synthèse de la pensée de l'écrivain, du moins jusqu'à l'époque de sa publication, car Glissant n'a jamais cessé, jusqu'à sa mort (2011), de se mettre à l'écoute du monde et de s'efforcer de lui donner une voix. On pourrait dire qu'il s'agit de deux ouvrages complémentaires qui ont eu la chance de paraître presque au même moment en Italie, au cours de l'année 2020, et dont la lecture peut constituer une bonne introduction à l'œuvre de Glissant, une œuvre puissante et chaotique (au sens positif que lui confère l'écrivain) qui a encore besoin d'un portulan pour des lecteurs nouveaux. Il faut avant tout souligner que cette nouvelle édition de l'Introduzione a una poetica del Diverso fait partie d'un projet de traduction de textes de la Caraibe, "Spin TransKarib» de l'Université Ca' Foscari de Venise, un projet élaboré par de jeunes chercheurs, ce qui laisse espérer une continuité pour les années à venir et donc la réalisation de nouvelles traductions glissantiennes.

Dans sa postface, Giuseppe Sofo, après avoir fait un rapide, et très utile, bilan de la réception de l'œuvre de Glissant en Italie, indique de façon détaillée les révisions les plus importantes apportées à la traduction de Francesca Neri, qui date désormais de plus de vingt ans. Le texte de 1998 avait opéré, on ne sait si par choix de la traductrice 
ou du directeur de la collection, deux interventions majeures sur le titre: le mot «introduction» en avait été éliminé et le complexe «Divers» glissantien, avec un D majuscule, était devenu un plus réductif «diverso». Et encore, en vue d'une simplification, peut-être nécessaire à l'époque, on avait éliminé, ou réduit, les questions qui accompagnaient chaque chapitre. Toutefois chaque chapitre étant à l'origine une conférence, un texte «oral», suivie d'un débat, il était important d'en préserver le ton et la dynamique, aussi bien que le concours de voix multiples. Giuseppe Sofo nous fournit aussi des renseignements sur les choix traductifs du texte dans d'autres langues et, de façon plus générale, sur les projets de diffusion de l'œuvre de Glissant dans le monde. Mais la postface ne se limite pas aux problèmes de traduction et de diffusion, elle se propose aussi une finalité herméneutique et propédeutique à la lecture du texte: une introduction à l'«Introduction», dont le mérite est bien synthétisé dans l'explicit: «Leggere i segni di Glissant, seguire la sua traccia significa infatti pensare con la sua opera, più che alla sua opera. Quello che nasce dalla lettura dello scrittore martinicano è un nuovo sguardo sulla realtà, e Introduzione a una poetica del Diverso ci permette di compiere un primo passo con questi nuovi occhi, prima di intraprendere un viaggio più lungo all'interno della sua vasta opera e nel tessuto della diversità del "Tutto-mondo" e del mondo tutto» (p. 148). 